\title{
Equivalence of partition properties and determinacy
}

(set theory/descriptive set theory/constructible from the reals universe)

\author{
Alexander S. Kechris and W. Hugh Woodin
}

Department of Mathematics, California Institute of Technology, Pasadena, California 91125

Communicated by Stephen C. Kleene, December 15, 1982

ABSTRACT It is shown that, within $L(\mathbb{R})$, the smallest inner model of set theory containing the reals, the axiom of determinacy is equivalent to the existence of arbitrarily large cardinals below $\Theta$ with the strong partition property $\kappa \rightarrow(\kappa)^{\kappa}$.

\section{STATEMENTS OF RESULTS}

Section 1.1. The axiom of determinacy (AD) is the assertion that every two-person perfect-information infinite game on the integers is determined. It has been proposed as a new strong axiom of set theory that the AD holds in the smallest inner model of ZF containing the set of reals $\mathbb{R}$, denoted by $L(\mathbb{R})$. An extensive theory of the structure of $L(\mathbb{R})$ has been developed over the years under the hypothesis that $L(\mathbb{R}) \vDash A D$, and it appears at this stage that $\mathrm{ZF}+\mathrm{AD}+\mathrm{V}=L(\mathbb{R})$ behaves as a "complete" theory of the inner model $L(\mathbb{R})$ in the same sense that $Z F+V$ $=\mathrm{L}$ appears as a "complete" theory of Gödel's constructible universe $\mathrm{L}$.

The study of the structure of $L(\mathbb{R})$ basically reduces to the study of two seemingly unrelated aspects: $(i)$ the "descriptive" set theoretic-i.e., the study of the sets of reals in $L(\mathbb{R})$ - and (ii) the "pure" set theoretic-i.e., the study of cardinals in $L(\mathbb{R})$. Since nothing novel happens above the cardinal $\Theta$ of the continuum-i.e., the supremum of the ordinals onto which we can map $\mathbb{R}$-it is enough to restrict ourselves to cardinals below $\Theta$.

One of the most fascinating phenomena discovered in the course of the study of $L(\mathbb{R})$ under the AD is the extremely close interrelationship that exists between the above two aspects. Over and over again, problems about the structure of cardinals are resolved by connecting them to problems about sets of reals and applying descriptive set theoretic methods. And vice versa, questions in descriptive set theory have been attacked by using set theoretic methods involving infinitary combinatorics, ultraproducts, etc.

The basic hypothesis in this study - namely, the AD - is clearly an assertion concerning the descriptive set theoretic element of $L(\mathbb{R})$. Our main result establishes a purely set theoretic equivalent formulation of this hypothesis. Before we state it, we recall the following standard notation from the theory of partition properties: For each set of ordinals $H$ and each ordinal $\lambda$, we let $[H]^{\lambda}$ be the set of all increasing $\lambda$ sequences from $H$. Now, for cardinals $\lambda \leq \kappa, \mu<\kappa, \kappa \rightarrow(\kappa)_{\mu}^{\lambda}$ means that, for every partition $F:[\kappa]^{\lambda} \rightarrow \mu$, there is a set $H \subseteq \kappa$ of cardinality $\kappa$ that is homogeneous for $F$; i.e., $F \backslash[H]^{\lambda}$ is constant. We say that $\kappa$ has the strong partition property if $\kappa \rightarrow(\kappa)_{\mu}^{\kappa}, \forall \mu<\kappa$.

We now have

THEOREM 1. Assume ZF + DC. Then, the following are equivalent.

(i) $L(\mathbb{R})=A D$;

The publication costs of this article were defrayed in part by page charge payment. This article must therefore be hereby marked "advertisement" in accordance with 18 U. S. C. \$1734 solely to indicate this fact. (ii) $L(\mathbb{R})=\forall \lambda<\Theta \exists \kappa(\kappa>\lambda \wedge \kappa$ has the strong partition property).

(One can also add to $i$ and $i$ the equivalent $i i i L(\mathbb{R}) \models \forall \lambda<\Theta$ $\exists \kappa\left[\kappa>\lambda \wedge \kappa \rightarrow(\kappa)_{2}^{\lambda}\right]$.

It has been already shown by Kechris $e$ t al. (1) that ZF + DC $+\mathrm{AD} \Rightarrow \forall \lambda<\Theta \exists \kappa(\kappa>\lambda \wedge \kappa$ has the strong partition property). The question of whether the converse also holds in $L(\mathbb{R})$ was also first explicitly stated in that paper.

As is customary in descriptive set theory, we identify the set of reals $\mathbb{R}$ with the space $\omega^{\omega}$ of all infinite sequences from $\omega=$ $\{0,1,2, \ldots\}$. Recall now that a set $A \subseteq \mathbb{R}$ is called $\lambda$-Souslin, for $\lambda$ an ordinal, if there is a tree $T$ on $\omega \times \lambda$ such that $A=p[T]$ $=\left\{\alpha \in \mathbb{R}: \exists f \in \lambda^{\omega} \forall n(\alpha \uparrow n, f \uparrow n) \in T\right\}$, and $A$ is called Souslin if it is $\lambda$-Souslin for some $\lambda$. (We follow here and below common terminology and notation in descriptive set theory; see ref. 2; in particular variables $\alpha, \beta, \gamma$, and $\delta$ vary always over $\mathbb{R})$. In ref. 1 , it is proved, again in ZF + DC, that if $\forall \lambda<\Theta \exists \kappa$ $\left[\lambda<\kappa \wedge \kappa \rightarrow(\kappa)_{2}^{\lambda}\right]$, then every Souslin set of reals is determined. Thus, Theorem 1 is an immediate consequence of

THEOREM 2. Assume ZF $+D C$. Then, the following are equivalent:

(i) $L(\mathbb{R})=A D$

(ii) $L(\mathbb{R})=$ every Souslin set of reals is determined.

The proof of Theorem 2 relies heavily on the work of Steel (3), which analyzes the propagation of the scale property in $L(\mathbb{R})$, using the fine structure of this inner model. Also, in the proof of Theorem 2 as well as of Theorems 3 and 4 below, essential use is made of a technique of Martin (4) for handling finite strings of alternating quantifiers over $\mathbb{R}$ via iterated products of the Martin measure on the Turing degrees.

There are several strengthenings and corollaries of the preceding theorems. For instance, in Theorem 2, we can weaken $i i$ as follows: Call a point class $\boldsymbol{\Gamma}$ reasonable if it is closed under $\wedge, \vee$, bounded number quantification, continuous substitutions and $\exists^{\mathbb{R}}$ or $\forall^{\mathbb{R}}$ and is $\mathbb{R}$ parametrized. We abbreviate by $\Gamma$ determinacy the statement that every set of reals in $\Gamma$ is determined. Then, we have that the following are equivalent in $\mathrm{ZF}+\mathrm{DC}$ :

(i) $L(\mathbb{R})=\mathrm{AD}$

(ii) $L(\mathbb{R})=$ for every reasonable $\Gamma$ with the scale property, $\Gamma$ determinacy holds.

The following is also an immediate corollary of Theorem 2. CoROllary. Assume ZF $+D C$. If there is a cardinal $\kappa$ with $\kappa \geq \Theta^{\mathrm{L}(\mathbf{R})}$ and $\kappa \rightarrow(\kappa)_{2}^{\kappa}$ or even $\kappa \rightarrow(\kappa)_{2}^{\lambda}, \forall \lambda<\Theta^{\mathrm{L}(\mathbb{R})}$, then $L(\mathbb{R})$ $=A D$. In particular,

$\operatorname{Con}\left(\mathrm{ZF}+D C+\exists \kappa \geq \Theta^{\mathrm{L}(\mathbb{R})}\left[\kappa \rightarrow(\kappa)_{2}^{\kappa}\right]\right)$

$$
\Rightarrow \operatorname{Con}(\mathrm{ZF}+\mathrm{DC}+\mathrm{AD}) \text {. }
$$

Note that in the above $\kappa$ is assumed to have the partition property in the universe, not necessarily in $L(\mathbb{R})$. Conceivably, this result could be used to demonstrate the consistency of ZF

Abbreviation: $\mathrm{AD}$, axiom of determinacy 
+ $\mathrm{DC}+\mathrm{AD}$ from appropriate large cardinal assumptions.

We describe next a corollary concerning the relativization of partition properties to $L(\mathbb{R})$.

COROLLARY. Let $\sigma$ be one of the statements (i) $\forall \lambda<\Theta \exists$ $\kappa\left[\kappa>\lambda \wedge \forall \mu<\kappa, \kappa \rightarrow(\kappa)_{\mu}^{\kappa}\right]$ or (ii) $\forall \lambda<\Theta \exists \kappa[\lambda>\kappa \wedge$ $\left.\kappa \rightarrow(\kappa)_{2}^{\lambda}\right]$. Then, assuming $Z F+D C$, $\sigma$ relativizes to $\mathrm{L}(\mathbb{P})$-i.e., $\sigma \Rightarrow \sigma^{\mathrm{L}(\mathbb{R})}$. Also, $\mathrm{L}(\mathbb{R})=\sigma \Rightarrow \forall \mu<\omega_{1}\left[\omega_{1} \rightarrow\left(\omega_{1}\right)_{\mu}^{\omega_{1}}\right]$.

Finally, we have the following rather curious reflection property of $L(\mathbb{R})$ : Assume $\mathrm{ZF}+\mathrm{DC}+\mathrm{V}=L(\mathbb{R})$. Let $\sigma_{0}=$ least stable (modulo $\mathbb{R})$ ordinal-i.e., the least $\xi$ with $L_{\xi}(\mathbb{R})<_{1} L(\mathbb{R})$. If $\kappa \rightarrow(\kappa)_{2}^{\sigma_{0}}$ for some $\kappa \geq \sigma_{0}$, then there are many small cardinals $\lambda<\kappa\left(\right.$ e.g., $\left.\lambda=\omega_{1}\right)$ satisfying the strong partition property and also arbitrarily large below $\Theta$ cardinals with the strong partition property.

Section 1.2. The key new tool in the proof of Theorem 2.(and thus Theorem 1) is a "transfer" theorem of the form (in ZF + DC) $\boldsymbol{\Gamma}_{1}$ determinacy $\Rightarrow \boldsymbol{\Gamma}_{2}$ determinacy, where $\boldsymbol{\Gamma}_{1}, \boldsymbol{\Gamma}_{2}$ are pointclasses with appropriate properties and interrelationships, and $\boldsymbol{\Gamma}_{2}$ is "much bigger" than $\boldsymbol{\Gamma}_{1}$. A basic instance of this type of theorem will be stated below after we establish some notation. Let, for each pointclass $\Gamma, \Sigma_{n}^{*}(\Gamma)$ be defined inductively on $n$ by letting

$\boldsymbol{\Sigma}_{1}^{*}(\boldsymbol{\Gamma})=$ all projections of Boolean combinations of sets in $\boldsymbol{\Gamma}$,

$$
\begin{aligned}
& \boldsymbol{\Pi}_{n}^{*}(\boldsymbol{\Gamma})=\text { all complements of sets in } \boldsymbol{\Sigma}_{n}^{*}(\boldsymbol{\Gamma}), \\
& \boldsymbol{\Sigma}_{n+1}^{*}(\boldsymbol{\Gamma})=\text { all projections of sets in } \boldsymbol{\Pi}_{n}^{*}(\boldsymbol{\Gamma}) .
\end{aligned}
$$

A typical for our purposes example of such a $\Gamma$ is the pointclass IND of all inductive sets on the structure of the continuum. In this case, we just write $\Sigma_{n}^{*}=\Sigma_{n}^{*}$ (IND), etc. We now have

THEOREM 3. Assume ZF + DC. Let $\Gamma$ be a pointclass closed under continuous substitutions, $\wedge, \vee, \exists^{\mathbb{R}}, \forall^{\mathbb{R}}$. If $\Gamma$ has the prewell-ordering and uniformization properties, then, for $\Delta=$ $\Gamma \cap \check{\Gamma}$ (the ambiguous part of $\Gamma$ ), we have $\Delta$ determinacy $\Rightarrow$ $\Sigma_{\mathrm{n}}^{*}(\Gamma)$ determinacy, $\forall \mathrm{n}$.

Corollary. Assume ZF + DC. Then,

$$
\text { HYP determinacy } \Rightarrow \Sigma_{\mathrm{n}}^{*} \text { determinacy, } \forall \mathrm{n} \text {, }
$$

where HYP = the class of hyperprojective sets.

Harrington (5) and D. A. Martin (personal communication) have shown in $\mathrm{ZF}+\mathrm{DC}$,

$$
\boldsymbol{\Pi}_{1}^{1} \text { determinacy } \Leftrightarrow\left(\boldsymbol{\omega} \cdot n-\Pi_{1}^{1}\right) \text { determinacy, } \forall n .
$$

Abbreviate for convenience

$$
\mathbf{M}_{n}=\omega \cdot n-\Pi_{1}^{1} \text {. }
$$

Let $\Theta$ be the game quantifier (on $\omega$ ) and for any point class $\Gamma$

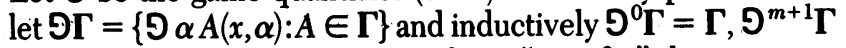
$=\Xi\left[\Xi^{m}(\Gamma)\right]$. Another instance of our "transfer" theorem provides a generalization of the Harrington-Martin result to all even levels of the projective hierarchy.

Theorem 4. Assume ZF + DC. Then, for $\mathrm{k} \geq 1$,

$$
\Delta_{2 \mathrm{k}}^{1} \text {. determinacy } \Leftrightarrow \mathrm{D}^{2 \mathrm{k}-1} \mathbf{M}_{\mathrm{n}} \text { determinacy, } \quad \forall \mathrm{n} \text {. }
$$

For instance, if $k=1$, this says that, in $\mathrm{ZF}+\mathrm{DC}$,

$$
\boldsymbol{\Delta}_{2}^{1} \text { determinacy } \Leftrightarrow \boldsymbol{D M}_{n} \text { determinacy, } \forall n \text {. }
$$

In particular, combining this result with the main theorem of Martin (6), we see that in ZFC,

$$
\exists \text { iterable } j: V_{\lambda} \rightarrow V_{\lambda} \Rightarrow \boldsymbol{S M}_{n} \text { determinacy, } \forall n \text {. }
$$

The classes $\mathbf{S M}_{n}$ are substantially bigger than $\Sigma_{2}^{1}$ but still well within $\mathbf{\Delta}_{3}^{\mathbf{l}}$.

Since $\left(\omega^{2}+1-\Pi_{1}^{1}\right.$ determinacy) proves the consistency of
$\Pi_{1}^{1}$ determinacy, the Harrington-Martin result is basically best possible. Similarly, Theorem 4 is basically best possible.

We do not know at this stage whether Theorem 4 extends to odd levels (bigger than 1) or whether our techniques can be used to give a different proof of the Harrington-Martin theoremthe only known proof goes through the theory of sharps while our methods are direct and "purely" descriptive theoretic.

\section{PROOF OF THEOREM 3}

We start with the proof of Theorem 3 , since it will be used in the proof of Theorem 2.

Assume $\Delta$ determinacy below. Let $A \subseteq \mathbb{R} \times \mathbb{R}$ be a $\Sigma_{n}^{*}(\Gamma)$ game, say $n=2$ for notational simplicity. Thus,

$$
A(\alpha, \beta) \Leftrightarrow \exists \gamma \forall \delta[R(\alpha, \beta, \gamma, \delta) \bigvee S(\alpha, \beta, \gamma, \delta)],
$$

where $R \in \Gamma$ and $S \in \check{\Gamma}$. Let

$$
P(i, x) \Leftrightarrow[i=0 \wedge R(x)] \bigvee[i \neq 0 \wedge \neg S(x)] .
$$

So $P \in \Gamma$. Let $\varphi: P \rightarrow \kappa$ be a $\Gamma$ norm on $P$. Note that $\kappa$ is limit and confinality $(\kappa)>\omega$, since otherwise $P$ is in $\Delta$ and there is nothing to prove. For $\xi<\kappa$, let $R_{\xi}(x) \Leftrightarrow \varphi(0, x)<\xi, S_{\xi}(x) \Leftrightarrow \neg$ $[\varphi(1, x)<\xi]$, so that $R=\cup_{\xi} R_{\xi}, S=\cap_{\xi} S_{\xi}$ and $\xi<\eta \Rightarrow R_{\xi} \subseteq$ $R_{\eta} \wedge S_{\xi} \supseteq S_{\eta}$, where in this section only ordinal variables $\xi, \eta$ vary over ordinals less than $\kappa$. Thus,

$$
A(\alpha, \beta) \Leftrightarrow \exists \gamma, \forall \delta\left[\exists \xi R_{\xi}(\alpha, \beta, \gamma, \delta) \bigvee \forall \xi S_{\xi}(\alpha, \beta, \gamma, \delta)\right] .
$$

Let $\sigma$ denote a strategy for player $1, \tau$ be a strategy for player $2, \sigma * \beta$ be the play of 1 following $\sigma$ when 2 plays $\beta$, and $\tau * \alpha$ be the play of 2 following $\tau$ when 1 plays $\alpha$. Then, assuming $A$ is not determined; toward a contradiction, we have

$$
\forall \sigma, \tau \exists \alpha, \beta[A(\alpha, \tau * \alpha) \wedge \neg A(\sigma * \beta, \beta)],
$$

or explicitly

$$
\begin{aligned}
\forall \sigma, \tau \exists \alpha, \beta\left\{\exists \gamma \forall \delta \left[\exists \xi R_{\xi}(\alpha, \tau * \alpha, \gamma, \delta)\right.\right. \\
\left.\vee \forall \xi S_{\xi}(\alpha, \tau * \alpha, \gamma, \delta)\right] \wedge \forall \gamma \exists \delta[\forall \xi \\
\left.\left.\neg R_{\xi}(\sigma * \beta, \beta, \gamma, \delta) \wedge \exists \xi \neg S_{\xi}(\sigma * \beta, \beta, \gamma, \delta)\right]\right\} .
\end{aligned}
$$

Below, we use the letters $c, d, e, \ldots$ to denote Turing degrees. Let $c \leq d$ be the usual partial ordering on the Turing degrees induced by the Turing reducibility $\gamma \leq_{T} \delta$ between reals, and let $\gamma \leq d$ mean $\gamma \leq_{T} \delta$ for any $\delta$ of degree $d$. Finally, abbreviate

$$
\begin{aligned}
\forall^{*} c P(c) & \Leftrightarrow \exists c_{0} \forall c \geq c_{0} P(c) \\
& \Leftrightarrow P(c) \text { holds on a cone of degrees. }
\end{aligned}
$$

Then, it is easy to check that

$$
\exists \gamma \forall . \delta P(\gamma, \delta) \Rightarrow \forall^{*} c \forall^{*} d \exists \gamma \leq c \forall \delta \leq d P(\gamma, \delta)
$$

and

$$
\forall \gamma \exists \delta P(\gamma, \delta) \Rightarrow \forall^{*} c \forall^{*} d \forall \gamma \leq c \exists \delta \leq d P(\gamma, \delta) .
$$

Using these formulas, we conclude from the preceding paragraph that the following holds:

$$
\begin{aligned}
\forall \sigma, \tau \exists \alpha, \beta\left\{\forall^{*} c \forall^{*} d(\exists \gamma \leq c \forall \delta\right. \\
\leq d\left[\exists \xi R_{\xi}(\alpha, \tau * \alpha, \gamma, \delta) \vee \forall \xi S_{\xi}(\alpha, \tau * \alpha, \gamma, \delta)\right] \wedge \forall \gamma \\
\leq c \exists \delta \leq d\left[\forall \xi \neg R_{\xi}(\sigma * \beta, \beta, \gamma, \delta)\right. \\
\left.\left.\left.\wedge \exists \xi \neg . S_{\xi}(\sigma * \beta, \beta, \gamma, \delta)\right]\right)\right\} .
\end{aligned}
$$


Call the formula inside $\{\cdots\} \Phi$.

Fix now a real $x \in \mathbb{R}$. By a simple Skolem-Löwenheim argument, find a countable set $M \subseteq \mathbb{R}$ that contains $x$, is closed under the pairing function $(x, y) \mapsto x \oplus y=\{x(0), y(0), x(1), y(1), \ldots\}$, is downward closed under $\leq_{T}$, and is such that $\forall \sigma, \tau \in M \exists$ $\alpha, \beta \in M\{\Phi\}$. Using the countability of $M$, the fact that the $R_{\xi}$ and $\neg S_{\xi}$ are increasing, and the fact that cofinality $(\kappa)>\omega$, we conclude by further simple manipulations that

$$
\forall^{*} c \forall^{*} d \exists \eta \forall \sigma, \tau \in M \exists \alpha, \beta \in M Q_{\eta}(\sigma, \tau, \alpha, \beta, c, d),
$$

where

$$
\begin{gathered}
Q_{\eta}(\sigma, \tau, \alpha, \beta, c, d) \Leftrightarrow \exists \gamma \leq c \forall \delta \\
\leq d\left[R_{\eta}(\alpha, \tau * \alpha, \gamma, \delta) \vee S_{\eta}(\alpha, \tau * \alpha, \gamma, \delta)\right] \wedge \forall \gamma \leq c \exists \delta \\
\leq d\left[\neg R_{\eta}(\sigma * \beta, \beta, \gamma, \delta) \wedge \neg S_{\eta}(\sigma * \beta, \beta, \gamma, \delta)\right] .
\end{gathered}
$$

Call a countable set $M \subseteq \mathbb{R} x$-good if it contains $x \in \mathbb{R}$, is closed under pairing, and is downward closed under $\leq_{T}$. Think of $M$ as a real in some appropriate coding. Then, if

$$
\begin{array}{r}
P(x, M) \Leftrightarrow M \text { is } x-\operatorname{good} \wedge \forall^{*} c \forall^{*} d \exists \eta \forall \sigma, \tau \in M \exists \alpha, \beta \\
\in M Q_{\eta}(\sigma, \tau, \alpha, \beta, c, d),
\end{array}
$$

$P$ is in $\Gamma$ and we have shown that $\forall x \exists M P(x, M)$. By the uniformization property of $\Gamma$, let $F: \mathbb{R} \rightarrow \mathbb{R}$ be in $\Delta$, such that, if $F(x)=M_{x}$, we have $P\left(x, M_{x}\right)$. Put $m(x)=\left\{M_{y}: y \leq_{T} x \wedge M_{y}\right.$ is $x-$ good\}. Then $\forall x \forall^{*} c \forall^{*} d \exists \eta \exists M \in m(x) \forall \sigma, \tau \in M \exists \alpha, \beta$ $\in M Q_{\eta}(\sigma, \tau, \alpha, \beta, c, d)$. Let $\mathscr{D}$ be the set of Turing degrees and define, for each real $x \in \mathbb{R}$, a partial function $f_{x}: \mathscr{D} \times \mathscr{D} \rightarrow \kappa$ by letting

$$
\begin{gathered}
f_{x}(c, d)=\text { least } \eta \text { such that } \\
\exists M \in m(x) \forall \sigma, \tau \in M \exists \alpha, \beta \in M Q_{\eta}(\sigma, \tau, \alpha, \beta, c, d) .
\end{gathered}
$$

Thus, $\forall x \forall^{*} c \forall^{*} d\left[f_{x}(c, d)\right.$ exists].

Consider now the following game $A^{*}$ : 1 plays $\alpha \oplus x_{0}$ and 2 plays $x_{1} \oplus \beta$. Let $x=x_{0} \oplus x_{1}$. Then 1 wins iff $\forall^{*} c \forall^{*} d \exists \gamma$ $\leq c \forall \delta \leq d\left[R_{f_{x}(c, d)}(\sigma, \beta, \gamma, \delta) \bigvee S_{f_{x}(c, d)}(\alpha, \beta, \gamma, \delta)\right]$. This is a game in $\Delta$, so it is determined. This will lead to a contradiction.

Say $\bar{\sigma}$ is a winning strategy for 1 (the proof is similar in case 2 wins). Notice first the following fact, easily proved by contradiction, using $\boldsymbol{\Delta}$ determinacy,

$$
\forall v \exists w \geq_{T} v \forall w^{\prime} \geq_{T} w \forall^{*} c \forall^{*} d\left[f_{w^{\prime}}(c, d) \geq f_{w}(c, d)\right] .
$$

Find then $w \geq_{T} \bar{\sigma}$ such that $\forall w^{\prime} \geq_{T} w \forall^{*} c \forall^{*} d\left[f_{w^{\prime}}(c, d) \geq\right.$ $f_{w}(c, d)$ ]. Consider then the strategy $\sigma_{0}$ for 1 in the original game $A$ given by $\sigma_{0} * \beta=[\bar{\sigma} *(w \oplus \beta)]_{0}$, where $z=(z)_{0} \oplus(z)_{1}$. Thus, if in the game $A^{*}, 2$ plays $w \oplus \beta, 1$ answers by $\left(\sigma_{0} * \beta\right) \oplus x_{0}$, for some $x_{0}$. Clearly, $\sigma_{0} \leq_{T} w$. By the definition of $f_{w}(c, d)$ and using $\Delta$ determinacy and the countability of $m(w)$, we have $\exists$ $M \in m(w) \forall^{*} c \forall^{*} d \forall \sigma, \tau \in M \exists \alpha, \beta \in M Q_{f_{w}(c, d)}(\sigma, \tau, \alpha, \beta, c, d)$, so fix $M_{0} \in m(w)$, witnessing this existential statement. Then, $M_{0}$ is $w$-good, so $\sigma_{0} \in M_{0}$. From the definition of $Q_{\eta}$, we then have some $\beta_{0} \in M_{0}$ with $\forall^{*} c \forall^{*} d \forall \gamma \leq c \exists^{\eta} \delta \leq d[\neg$ $\left.R_{f_{w}(c, d)}\left(\sigma_{0} * \beta_{0}, \beta_{0}, \gamma, \delta\right) \wedge \neg S_{f_{w}(c, d)}\left(\sigma_{0} * \beta_{0}, \beta_{0}, \gamma, \delta\right)\right]$. Put $\alpha_{0}=$ $\sigma_{0} * \beta_{0}$. Then, for some $x_{0}, \alpha_{0} \oplus x_{0}$ with $w \oplus \beta_{0}$ is a run of the game $A^{*}$ in which 1 follows $\bar{\sigma}$. Thus, if $x=x_{0} \oplus w$, we have $\forall^{*}$ c $\forall^{*} \mathrm{~d} \exists \gamma \leq c \forall \boldsymbol{\delta} \leq d\left[\mathbb{R}_{f_{x}(c, d)}\left(\alpha_{0}, \beta_{0}, \gamma, \delta\right) \vee S_{f_{x}(c, d)}\right.$ $\left.\left(\alpha_{0}, \beta_{0}, \gamma, \delta\right)\right]$. Thus, we have a contradiction if we can show that $\forall^{*} c \forall^{*} d\left[f_{x}(c, d)=f_{w}(c, d)\right]$. First, by the choice of $w$ and the fact that $w \leq_{T} x$, we have that $\forall^{*} c \forall^{*} d\left[f_{w}(c, d) \leq f_{x}(c, d)\right]$. Now, $M_{0}$ is $w$-good and $\sigma_{0}, \beta_{0} \in M_{0}$ so that $\alpha_{0}, x_{0}, x \in M_{0}$. Thus, $M_{0}$ is $x$-good. Since $M_{0} \in m(w), M_{0}=M_{y}$ for some $y \leq_{T} w \leq_{T} x$ and thus $M_{0} \in m(x)$. By the choice of $M_{0}$, then we have that $\forall^{*} c$ $\forall^{*} d \exists M \in m(x) \forall \sigma, \tau \in M \exists \alpha, \beta \in M Q_{f_{w}(c, d)}(\sigma, \tau, \alpha, \beta, c, d)$.
So, from the definition of $f_{x}$, we have that $\forall^{*} c \forall^{*} d\left[f_{x}(c, d) \leq\right.$ $f_{w}(c, d)$ ], which completes our proof.

Remark. The proof of Theorem 4 is similar to the above, starting from the following representation of $5 M_{n}$ sets (see ref. 4):

$$
x \in A \Leftrightarrow L[x] \vDash \varphi\left(x, u_{1}, \ldots, u_{n}\right),
$$

where $\varphi$ is a formula and $u_{1}, u_{2}, \ldots$ are the uniform indiscernibles.

\section{PROOF OF THEOREM 2}

Assume $\mathrm{ZF}+\mathrm{DC}+\mathrm{V}=L(\mathbb{R})$ and "Every Souslin set of reals is determined." We will prove by induction on $\xi \in$ ORD that every set in $J_{\xi}(\mathbb{R})$ is determined, where $\left\{J_{\xi}(\mathbb{R}): \xi \in\right.$ ORD $\}$ is the Jensen hierarchy for $L(\mathbb{R})$ (see ref. 3). Abbreviate this by $J_{\xi}(\mathbb{R})$ determinacy. So assume $J_{\xi}(\mathbb{R})$ determinacy to prove $J_{\xi+1}(\mathbb{R})$ determinacy. Assume also that $\exists A \subseteq \mathbb{R}\left[A \in J_{\xi+1}(\mathbb{R})-J_{\xi}(\mathbb{P})\right]$.

We will need here the following concept from Steel (3): An interval of ordinals $[\eta, \zeta]$ where $\eta \leq \zeta$ is called a $\Sigma_{1}$ gap if $(i)$ $J_{\eta}(\mathbb{R})<{ }_{1}^{\mathbb{R}} J_{\zeta}(\mathbb{R}),(i i) \forall \eta^{\prime}<\eta J_{\eta^{\prime}}(\mathbb{R}) K_{1}^{\mathbb{R}} J_{\eta}(\mathbb{R})$, and $(i i i) \forall \zeta^{\prime}>\zeta$ $J_{\zeta}(\mathbb{R}) K_{1}^{\mathbb{R}} J_{\zeta}(\mathbb{R})$, where $M<_{n}^{X} N$ means that $M$ is a $\Sigma_{n}$ elementary substructure of $N$ when only parameters from $\mathrm{X} \cup\{\mathbb{R}\}$ are allowed. In particular, $M<_{n} N \Leftrightarrow M<_{n}^{M} N$. We also think of $\left[\sigma_{0}, \infty\right)$ as a $\Sigma_{1}$ gap, where $\sigma_{0}=$ least $\mathbb{R}$ stable $=$ least $\sigma$ such that $J_{\sigma}(\mathbb{R})$ $<_{1}^{\mathbb{R}} \mathrm{L}(\mathbb{R})$. By Steel (ref. 3, section 2.3) the $\Sigma_{1}$ gaps partition ORD. So let $[\eta, \zeta]$ be the $\Sigma_{1}$ gap in which $\xi$ belongs. We now consider various cases. We assume throughout that $\xi>1$. (The case $\xi=$ 1 is easily handled separately by the method of subcase 1.1 below.)

Case 1. $\xi=\eta$-i.e., $\xi$ is the beginning of a gap. Let then $A$ $\subseteq \mathbb{R}$ be a set in $J_{\xi+1}(\mathbb{R})$, say $A \in \mathbf{\Pi}_{3}\left[J_{\xi}(\mathbb{R})\right]$, for notational simplicity. Then, for some $\varphi \in \Sigma_{0}$ and some $z_{0} \in J_{\xi}(\mathbb{R}), \alpha \in A \Leftrightarrow$ $J_{\xi}(\mathbb{R})=\forall x_{0} \exists x_{1} \forall x_{2} \varphi\left(\alpha, x_{0}, x_{1}, x_{2}, z_{0}\right)$. Since, according to Steel (ref. 3, section 1.11), there is a partial $\Sigma_{1}\left[J_{\xi}(\mathbb{R})\right]$ map from $\mathbb{R}$ onto $J_{\xi}(\mathbb{R})$, we have that, for some $\psi_{i} \in \Sigma_{1}, \chi_{i} \in \Pi_{1}$, and $\delta_{0} \in \mathbb{R}$

$$
\begin{array}{r}
\alpha \in A \Leftrightarrow J_{\xi}(\mathbb{R}) \models \forall \beta_{0} \exists \beta_{1} \forall \beta_{2} V_{i=1}^{k}\left[\psi_{i}\left(\alpha, \beta_{0}, \beta_{1}, \beta_{2}, \delta_{0}\right)\right. \\
\left.\wedge \chi_{i}\left(\alpha, \beta_{0}, \beta_{1}, \beta_{2}, \delta_{0}\right)\right] .
\end{array}
$$

(We adopt here and below the convention that $\Sigma_{n}$ or $\Pi_{n}$ formulas for $n \geq 0$ are allowed to contain a constant for the set of reals R.)

Let $\Gamma$ be the point class of all sets of reals that are $\Sigma_{1}\left[J_{\xi}(\mathbb{R})\right]$. According to Steel (ref. 3, section 2.4), $\Gamma$ has the scale property, using $J_{\xi}(\mathbb{R})$ determinacy. We consider now two subcases on $\xi$.

Subcase 1.1. $J_{\xi}(\mathbb{R})$ is not admissible. Then, by Steel (ref. 3, section 2.5), it follows immediately that $A$ is in the class $\forall^{\mathbb{R}} \exists^{\mathbb{R}}$ $\forall^{\mathbb{R}} \Gamma$. But $\Gamma$ is closed under continuous substitutions $\wedge, \vee, \exists^{\mathbb{R}}$ and has the scale property so by the second periodicity theorem of Moschovakis (2), $\forall^{\mathbb{R}} \Gamma$ has the scale property, granting $\Gamma$ determinacy, which is given to us by the fact that every $\Gamma$ set is Souslin. Applying second periodicity twice more, we conclude that $\forall^{\mathbb{R}} \exists^{\mathbb{R}} \forall^{\mathbb{R}} \Gamma$ has the scale property, so $A$ is Souslin thus determined.

Subcase 1.2. $J_{\xi}(\mathbb{R})$ is admissible. Then, $\Gamma$ is closed under continuous substitutions $\wedge, \vee, \exists^{\mathbb{R}}, \forall^{\mathbb{R}}$ and has the scale property, so in particular the prewellordering and uniformization properties. Thus, by theorem 2 and the fact that $\Gamma$ determinacy holds, we have that $\mathbf{\Sigma}_{n}^{*}(\Gamma)$ determinacy holds for each $n$. But clearly $A$ $\in \Pi_{3}^{*}(\Gamma)$, so $A$ is determined.

Case 2. $\eta \leq \xi<\zeta$. Then, if $A \in J_{\xi+1}(\mathbb{R})$ is not determined, we have that $J_{\zeta}(\mathbb{R}) \models \exists X \subseteq \mathbb{R}\left(\mathrm{X}\right.$ is not determined), so $J_{\eta}(\mathbb{R})$ $\vDash \exists X \subseteq \mathbb{R}(\mathbf{X}$ is not determined $)$. But $J_{\eta}(\mathbb{R})$ determinacy holds, so we get a contradiction.

Case 3. $\eta<\xi=\zeta$. Let $\Gamma$ be the point class of all sets of reals 
that are $\Sigma_{1}$ over $J_{\eta}(\mathbb{R})$ with only real parameters. Since $[\eta, \xi]$ is a $\Sigma_{1}$ gap, it follows immediately that $\Gamma$ is closed under continuous substitutions $\wedge, \vee, \exists^{\mathbb{R}}, \forall^{\mathbb{R}}$ and has the scale property by theorem 2.2. of Steel (3). Also, let $n \geq 1$ be the least integer such that there is a $\Sigma_{n}\left[J_{\xi}(\mathbb{R})\right]$ set of reals that is not in $J_{\xi}(\mathbb{R})$. Assume also, toward a contradiction that there exists an undetermined game $A \in J_{\xi+1}(\mathbb{R})$, say for notational simplicity, it is $\Sigma_{n+2}\left[J_{\xi}(\mathbb{R})\right]$. Thus, for some $\psi \in \Sigma_{n}, z_{0} \in J_{\xi}(\mathbb{R}), A(\alpha, \beta) \Leftrightarrow J_{\xi}(\mathbb{R}) \models \exists x \forall$ $y \psi\left(\alpha, \beta, x, y, z_{0}\right)$. Since there is a partial map from $\mathbb{R}$ onto $J_{\xi}(\mathbb{R})$, which is $\Sigma_{n}\left[J_{\xi}(\mathbb{R})\right]$, we can transform this to $A(\alpha, \beta) \Leftrightarrow J_{\xi}(\mathbb{R})$ $=\exists \gamma \forall \delta V_{i=1}^{k}\left[\psi_{i}^{\prime}(\alpha, \beta, \gamma, \delta, w) \wedge \chi_{i}^{\prime}(\alpha, \beta, \gamma, \delta, w)\right]$, where $\psi_{i}^{\prime} \in$ $\Sigma_{n}, \chi_{i}^{\prime} \in \Pi_{n}$, and $w \in J_{\xi}(\mathbb{R})$.

Finally, since there is a $\Sigma_{1}$ map from $[\omega \xi]^{<\omega}=\{F \subset \omega \xi: F$ is finite $\times \mathbb{R}$ onto $J_{\xi}(\mathbb{R})$, we can put this in the form $A(\alpha, \beta) \Leftrightarrow$ $J_{\xi}(\mathbb{R})=\exists \gamma \forall \delta V_{i=1}^{k}\left[\psi_{i}\left(\alpha, \beta, \gamma, \delta, \delta_{0}, F_{0}\right) \wedge \chi_{i}\left(\alpha, \beta, \gamma, \delta, \delta_{0}, F_{0}\right)\right]$, where $\delta_{0} \in \mathbb{R}$ and $F_{0} \in[\omega \xi]^{<\omega}$.

Let us abbreviate

$$
\begin{aligned}
R_{\xi^{\prime}, F^{\prime}}(\alpha, \beta, \gamma, \delta) & \Leftrightarrow J_{\xi^{\prime}}(\mathbb{R}) \\
& =V_{i=1}^{k}\left[\psi_{i}\left(\alpha, \beta, \gamma, \delta, \delta_{0}, F^{\prime}\right) \wedge \chi_{i}\left(\alpha, \beta, \gamma, \delta, \delta_{0}, F^{\prime}\right)\right],
\end{aligned}
$$

for $\xi^{\prime} \leq \xi, F^{\prime} \in\left[\omega \xi^{\prime}\right]^{<\omega}$. Since $A$ is not determined, we have as in Section 2 that $\forall x \exists M\left\{M\right.$ is $x$-good $\wedge \forall^{*} c \forall^{*} d \forall \sigma, \tau \in$ $M \exists \alpha, \beta \in M\left[\exists \gamma \leq c \forall \delta \leq d R_{\xi, F_{0}}(\alpha, \tau * \alpha, \gamma, \delta) \wedge \forall \gamma \leq c\right.$ $\left.\left.\exists \delta \leq d \neg R_{\xi, F_{0}}(\sigma * \beta, \gamma, \delta)\right]\right\}$. We now consider two subcases. Subcase 3.1. The following holds:

$\forall x \exists M\left\{M\right.$ is $x-\operatorname{good} \wedge \forall^{*} c \forall^{*} d \exists \xi^{\prime}<\xi \exists F^{\prime} \in\left[\omega \xi^{\prime}\right]^{<\omega}$

$\forall \sigma, \tau \in M \exists \alpha, \beta \in M[\exists \gamma \leq c \forall \delta$

$$
\begin{aligned}
& \leq d R_{\xi^{\prime}, F^{\prime}}\left(\alpha, \tau^{*} \alpha, \gamma, \delta\right) \wedge \forall \gamma \leq c \exists \delta \\
&\left.\left.\leq d \neg R_{\xi^{\prime}, F^{\prime}}(\sigma * \beta, \beta, \gamma, \delta)\right]\right\} .
\end{aligned}
$$

Since the expression $P(x, M)$ following $\forall x \exists M$ is $\Sigma_{1}\left[J_{\xi}(\mathbb{R})\right]$ with real parameters only and $[\eta, \xi]$ is a $\Sigma_{1}$ gap, $P$ is in $\Gamma$, so by the uniformization property for $\Gamma$, we can find $F: \mathbb{R} \rightarrow \mathbb{R}$ in $\Delta$ such that, if $F(x)=M_{x}$, then $P\left(x, M_{x}\right)$. Define $m(x)$ as in Section 2 . Then abbreviating by $Q_{\xi^{\prime}, F^{\prime}}(\sigma, \tau, \alpha, \beta, c, d)$, the expression following $\forall \sigma, \tau \in M \exists \alpha, \beta \in M$ in the preceding formula, we have $\forall x \forall^{*} d \forall^{*} c \exists \xi^{\prime}<\xi \exists F^{\prime} \in\left[\omega \xi^{\prime}\right]^{<\omega} \exists M \in m(x) \forall \sigma, \tau \in$ $M \exists \alpha, \beta \in M Q_{\xi^{\prime}, F^{\prime}}(\sigma, \tau, \alpha, \beta, c, d)$. Since $[\eta, \xi]$ is a $\Sigma_{1}$ gap, we also have $\forall x \forall^{*} c \forall^{*} d \exists \xi^{\prime}<\eta \exists F^{\prime} \in\left[\omega \xi^{\prime}\right]^{<\omega} \exists M \in m(x)$ $\forall \sigma, \tau \in M \exists \alpha, \beta \in M Q_{\xi^{\prime}, F^{\prime}}(\sigma, \tau, \alpha, \beta, c, d)$. Now, let $f_{x}^{0}(c, d)=$ least $\xi^{\prime}<\eta \exists F^{\prime} \in\left[\omega \xi^{\prime}\right]^{<\omega} \exists M \in m(x) \forall \sigma, \tau \in M \exists \alpha, \beta \in$ $M Q_{\xi^{\prime}, F^{\prime}}(\sigma, \tau, \alpha, \beta, c, d), f_{x}^{1}(c, d)=$ the Kleene-Brouwer least $F^{\prime}$ $\in\left[\omega \cdot f_{x}^{0}(c, d)\right]^{<\omega}$ such that $\exists M \in m(x) \forall \sigma, \tau \in M \exists \alpha, \beta \in M$ $Q_{f_{x}^{o}(c, d), F^{\prime}}(\sigma, \tau, \alpha, \beta, c, d)$. Again, $\forall^{*} c \forall^{*} d\left[f_{x}^{i}(c, d)\right.$ is defined], $i$ $=0,1$. Consider now the game in which 1 plays $\alpha \oplus x_{0}$ and 2 plays $x_{1} \oplus \beta$ and, if $x=x_{0} \oplus x_{1}$, then 1 wins iff $\forall^{*} c \forall^{*} d \exists$ $\gamma \leq c \forall \delta \leq d R_{f_{x}^{q}(c, d), f_{x}^{\prime}(c, d)}(\alpha, \beta, \gamma, \delta)$. This is a $\Delta$ game so it is determined since every set in $\Gamma$ is Souslin and a contradiction is obtained exactly as in Section 2.

Subcase 3.2. Subcase 3.1. fails. Then, find $x_{0} \in \mathbb{R}$ such that, for all $x_{0}-\operatorname{good} M$, we have $\neg \forall^{*} c \forall^{*} d \exists \xi^{\prime}<\xi \exists F^{\prime} \in\left[\omega \xi^{\prime}\right]^{<\omega}$ $\forall \sigma, \tau \in M \exists \alpha, \beta \in M Q_{\xi, F^{\prime}}(\sigma, \tau, \alpha, \beta, c, d)$. From the formula preceding Subcase 3.1., it follows that we can find $M_{0}$, which is $x_{0}$-good, and $(i) \forall^{*} c \forall^{*} d \forall \sigma, \tau \in M_{0} \exists \alpha, \beta \in M_{0} Q_{\xi, F_{0}}$ $(\sigma, \tau, \alpha, \beta, c, d)$. Thus, also $\neg \forall^{*} c \forall^{*} d \exists \xi^{\prime}<\xi \exists F^{\prime} \in\left[\omega \xi^{\prime}\right]^{<\omega}$ $\forall \sigma, \tau \in M_{0} \exists \alpha, \beta \in M_{0} Q_{\xi^{\prime}, F^{\prime}}(\sigma, \tau, \alpha, \beta, c, d)$. Since the expression following $\neg \forall^{*} c \forall^{*} d$ is $\Sigma_{1}$ over $J_{\xi}(\mathbb{R})$ with only real parameters, it is in $\boldsymbol{\Gamma}$. But we have determinacy of all $\boldsymbol{\Gamma}$ Turing games, so we can conclude that (ii) $\forall^{*} c \forall^{*} d \forall \xi^{\prime}<\xi \forall F^{\prime} \in$ $\left[\omega \xi^{\prime}\right]^{<\omega} \exists \sigma, \tau \in M_{0} \forall \alpha, \beta \in M_{0} \neg Q_{\xi, F^{\prime}}(\sigma, \tau, \alpha, \beta, c, d)$. From $i$ and $i i$, we find $c_{0}, d_{0} \in \mathscr{D}$ with $\forall \sigma, \tau \in M_{0} \exists \alpha, \beta \in M_{0}$ $Q_{\xi, F_{0}}(\sigma, \tau, \alpha, \beta, c, d)$ and $\forall \xi^{\prime}<\xi \forall F^{\prime} \in\left[\omega \xi^{\prime}\right]^{<\omega} \exists \sigma, \tau \in M_{0} \forall$ $\alpha, \beta \in M_{0} \neg Q_{\xi, F^{\prime}}(\sigma, \tau, \alpha, \beta, c, d)$. From this and the definition of $Q_{\xi, F^{\prime}}$, it follows that there is a $\in J_{\xi}(\mathbb{R})$ such that the $\Sigma_{n}$ type realized by $a$ in $J_{\xi}(\mathbb{R})$ is not realized in any $J_{\xi^{\prime}}(\mathbb{R})$, with $\xi^{\prime}<\xi$ where for $b \in J_{\xi}(\mathbb{R})$ the $\Sigma_{n}$ type realized by $b$ in $J_{\xi}(\mathbb{R})$ is $\{\vartheta(v): \vartheta$ is $\Sigma_{n}$ or $\Pi_{n}$ and $\left.J_{\xi}(\mathbb{R})=\vartheta(b)\right\}$. In the terminology of Steel (3), this means that $[\eta, \xi]$ is a weak $\Sigma_{1}$ gap. So by theorem 3.7 of Steel, we have that the point class $\Sigma$ of sets of reals that are $\Sigma_{n}\left[L_{\xi}(\mathbb{R})\right]$ has the scale property and moreover, by theorem 3.8 of Steel, every set of reals that is $\Pi_{n}\left[L_{\xi}(\mathbb{R})\right]$ is in the class $\forall^{\mathbb{R}} \Sigma$. So our undetermined set $A$ is in $\exists^{\mathbb{R}} \forall^{\mathbb{R}} \mathbf{\Sigma}$. Since $\Sigma$ is closed under continuous substitutions $\wedge, \vee, \exists^{\mathbb{R}}$, two applications of second periodicity lead to the conclusion that $A$ is Souslin, thus determined, which is a contradiction, and the proof of Theorem 2 is complete.

This research was partially supported by National Science Foundation Grant MCS 81-17804. A.S.K. is an A. P. Sloan Foundation Fellow.

1. Kechris, A. S., Kleinberg, E. M., Moschovakis, Y. N. \& Woodin, W. H. (1981) in Cabal Seminar 77-79, Lecture Notes in Mathematics, No. 839, eds. Kechris, A. S., Martin, D. A. \& Moschovakis, Y. N. (Springer, Berlin), pp. 75-100.

2. Moschovakis, Y. N. (1980) Descriptive Set Theory (North Holland, Amsterdam).

3. Steel, J. R. (1983) in Cabal Seminar 79-81, Lecture Notes in Mathematics, eds. Kechris, A. S., Martin, D. A. \& Moschovakis, Y. N. (Springer, Berlin), in press.

4. Martin, D. A. (1983) in Cabal Seminar 79-81, Lecture Notes in Mathematics, eds. Kechris, A. S., Martin, D. A. \& Moschovakis, Y. N. (Springer, Berlin), in press.

5. Harrington, L. A. (1978) J. Symb. Logic 43, 685-693.

6. Martin, D. A. (1980) in Proceedings of the International Congress of Mathematicians 1978, ed. Lehto, O. (Academia Scientiarum Fennica, Helsinki), pp. 269-273. 